\title{
EVOLUTION OF CRUSTAL MAGNETIC FIELDS IN ISOLATED NEUTRON STARS : COMBINED EFFECTS OF COOLING AND CURVATURE OF SPACE-TIME
}

\author{
SUJAN SENGUPTA \\ Mehta Research Institute of Mathematics and Mathematical Physics \\ Chhatnag Road, Jhusi, Allahabad 211 019, India. E-mail: sujan@mri.ernet.in
}

Received __; accepted _ 


\begin{abstract}
The ohmic decay of magnetic fields confined within the crust of neutron stars is considered by incorporating both the effect of neutron star cooling and the effect of space-time curvature produced by the intense gravitational field of the star. For this purpose a stationary and static gravitational field has been considered with the standard as well as the accelerated cooling models of neutron stars. It is shown that general relativistic effect reduces the magnetic field decay rate substantially. At the late stage of evolution when the field decay is mainly determined by the impurity-electron scattering, the effect of space-time curvature suppresses the role of the impurity content significantly and reduces the decay rate by more than an order of magnitude. Even with a high impurity content the decay rate is too low to be of observational interest if the accelerated cooling model along with the effect of space-time curvature is taken into account. It is, therefore, pointed out that if a decrease in the magnetic field strength by more than two orders of magnitude from its initial value is detected by observation then the existence of quark in the core of the neutron star would possibly be ruled out.
\end{abstract}

Subject headings: magnetic fields — relativity — stars : neutron 


\section{INTRODUCTION}

The magnetic field evolution in neutron stars has been the subject of much discussion over the years both in the observational and in the theoretical context. Recent observations of pulsars and their statistical analysis seem to imply that isolated pulsars do not undergo significant magnetic field decay during their life times (Wakatsuki et al 1992). The possible physical processes through which the magnetic field in the crust of isolated neutron stars may decay are ambipolar diffusion, Hall drift, Ohmic dissipation, etc. The reviews of Lamb (1991), Chanmugam (1992) and Phinney \& Kulkarni (1994) provide a reasonable account of our present understanding on the decay of magnetic fields in isolated neutron stars.

Sang \& Chanmugam (1987) demonstrated that the Ohmic decay of the dipole magnetic fields is not exponential. Considering magnetic field configurations which are initially confined to a small part of the crust and which vanish in the stellar core, several authors (Chanmugam \& Sang 1989; Urpin \& Muslimov 1992; Urpin \& Van Riper 1993) found that the relatively low electrical conductivity of the crustal matter causes the decay times too short to be of observational interest if the impurity concentration is high. On the other hand, Pethic \& Sahrling (1995) showed that if the field does not vanish in the core then the shortest possible decay time becomes about two orders of magnitude larger than the characteristic time-scale for decay when the field vanishes in the core. Bhattacharya \& Datta (1996) have studied the ohmic diffusion of magnetic flux expelled from the super-conducting core to the crust owing to spindown-induced field evolution and claimed that the result obtained by them is in agreement with the ohmic time-scale that appears to be required to explain the observed magnetic field strengths of isolated and binary neutron stars in the spindown-induced magnetic flux expulsion scenario.

Recently Sengupta (1997) demonstrated the significant contribution of space-time curvature in reducing the decay rate of the crustal magnetic fields in isolated neutron star 
by assuming a spherically symmetric stationary gravitational field. However, the cooling of neutron star has not been incorporated in that work for the sake of simplicity.

In this paper the Ohmic dissipation of the crustal magnetic field has been investigated by incorporating both the effects of neutron star cooling and the space-time curvature produced by the intense gravitational field of the star. The paper is organized in the following ways: in Section 2, the relevant equations are presented. In Section 3, the adopted neutron star model, the initial configurations of the magnetic field and the electrical conductivity have been discussed. The results are outlined in Section 4 and finally in Section 5 specific conclusions are made.

\section{MAGNETIC FIELD EVOLUTION IN CURVED SPACE-TIME}

Assuming hydrodynamic motions to be negligible and the anisotropy of the electrical conductivity of the crustal material is small, the induction equation in flat space-time can be written as:

$$
\frac{\partial \mathbf{B}}{\partial t}=-\nabla \times\left(\frac{c^{2}}{4 \pi \sigma} \nabla \times \mathbf{B}\right),
$$

where $\sigma$ is the electrical conductivity.

The covariant form of Maxwell's equations are given by:

$$
\frac{1}{\sqrt{-g}} \frac{\partial}{\partial x^{\nu}}\left(\sqrt{-g} F^{\mu \nu}\right)=-\frac{4 \pi}{c} J^{\mu}
$$

and

$$
\frac{\partial F^{\mu \nu}}{\partial x^{\lambda}}+\frac{\partial F^{\nu \lambda}}{\partial x^{\mu}}+\frac{\partial F^{\lambda \mu}}{\partial x^{\nu}}=0
$$


The generalized Ohm's law can be written as :

$$
J^{\mu}=\sigma g^{\mu \nu} F_{\nu \lambda} u^{\lambda}
$$

where $F_{\mu \nu}$ are the components of the electro-magnetic field tensor, $J^{\mu}$ are the components of the four-current density, $u^{\mu}$ are the components of the four velocity of the fluid, $g_{\mu \nu}$ are the components of space-time metric that describes the background geometry and $g=\operatorname{det}\left|g_{\mu \nu}\right|$. Here and afterwards Latin indices run over spatial co-ordinates only whereas Greek indices run over both time and space co-ordinates.

If a stationary gravitational field is taken into account, then using equations (2), (3) and (4) (neglecting the displacement current and taking $u^{i}=0$ ) the corresponding induction equation in curved space-time can be derived as:

$$
\frac{\partial F_{k j}}{\partial x^{0}}=\frac{\partial}{\partial x^{k}}\left[\frac{c}{4 \pi} \frac{1}{\sqrt{-g}} g_{\mu j} \frac{1}{\sigma u^{0}} \frac{\partial}{\partial x^{i}}\left(\sqrt{-g} F^{\mu i}\right)\right]-\frac{\partial}{\partial x^{j}}\left[\frac{c}{4 \pi} \frac{1}{\sqrt{-g}} g_{\mu k} \frac{1}{\sigma u^{0}} \frac{\partial}{\partial x^{i}}\left(\sqrt{-g} F^{\mu i}\right)\right]
$$

For the description of the background geometry I consider the exterior Schwarzschild metric which is given by

$$
d s^{2}=\left(1-\frac{2 m}{r}\right) c^{2} d t^{2}-\left(1-\frac{2 m}{r}\right)^{-1} d r^{2}-r^{2}\left(d \theta^{2}+\sin ^{2} \theta d \phi^{2}\right),
$$

where $m=M G / c^{2}, M$ being the total gravitational mass of the core. The justification for adopting the exterior Schwarzschild metric is provided in Sengupta (1997). Since, the crust consists of less than a few percent of the total gravitational mass, $M$ can be regarded as the total mass of the star. The non-zero components of the orthonormal tetrad $\lambda_{(\alpha)}^{\gamma}$ of the local Lorentz frame for the Schwarzschild geometry is given by

$$
\lambda_{(t)}^{t}=\left(1-\frac{2 m}{r}\right)^{-1 / 2}, \lambda_{(r)}^{r}=\left(1-\frac{2 m}{r}\right)^{1 / 2}, \lambda_{(\theta)}^{\theta}=1 / r, \lambda_{(\phi)}^{\phi}=1 / r \sin \theta .
$$

If $F_{(\alpha \beta)}$ are the components of the electro-magnetic field tensor in a local Lorentz frame, then the components of the electro-magnetic field tensor $F_{\gamma \delta}$ are defined in the 
curved space-time through the relation:

$$
F_{(\alpha \beta)}=\lambda_{(\alpha)}^{\gamma} \lambda_{(\beta)}^{\delta} F_{\gamma \delta}
$$

Using the metric given in equation (6), equation (5) can be reduced to

$$
\frac{\partial F_{k j}}{\partial x^{0}}=\frac{c}{4 \pi}\left[\frac{\partial}{\partial x^{k}}\left\{\frac{1}{r^{2} \sin \theta} g_{l j} \frac{1}{\sigma u^{0}} \frac{\partial}{\partial x^{i}}\left(r^{2} \sin \theta F^{l i}\right)\right\}-\frac{\partial}{\partial x^{j}}\left\{\frac{1}{r^{2} \sin \theta} g_{l k} \frac{1}{\sigma u^{0}} \frac{\partial}{\partial x^{i}}\left(r^{2} \sin \theta F^{l i}\right)\right\}\right] .
$$

Following the convention, I consider the decay of a dipolar magnetic field which has axial symmetry so that the vector potential $\mathbf{A}$ may be written as $\left(0,0, A_{\phi}\right)$ in spherical polar co-ordinates where $A_{\phi}=A(r, \theta, t)$. Since the hydrodynamic motion is negligible so $u^{i}=d x^{i} / d s=0$ and the metric gives

$$
u^{0}=\left(1-\frac{2 m}{r}\right)^{-1 / 2} .
$$

Therefore, from equation (8) we obtain

$$
\frac{\partial F_{r \phi}}{\partial t}=\frac{c^{2}}{4 \pi} \frac{\partial}{\partial r}\left[\frac{1}{\sigma}\left(1-\frac{2 m}{r}\right)^{1 / 2} \sin \theta\left\{\frac{\partial}{\partial r}\left[\left(1-\frac{2 m}{r}\right) \frac{1}{\sin \theta} F_{r \phi}\right]+\frac{\partial}{\partial \theta}\left(\frac{1}{r^{2} \sin \theta} F_{\theta \phi}\right)\right\}\right]
$$

and

$$
\frac{\partial F_{\theta \phi}}{\partial t}=\frac{c^{2}}{4 \pi} \frac{\partial}{\partial \theta}\left[\frac{1}{\sigma}\left(1-\frac{2 m}{r}\right)^{1 / 2} \sin \theta\left\{\frac{\partial}{\partial r}\left[\left(1-\frac{2 m}{r}\right) \frac{1}{\sin \theta} F_{r \phi}\right]+\frac{\partial}{\partial \theta}\left(\frac{1}{r^{2} \sin \theta} F_{\theta \phi}\right)\right\}\right] .
$$

Using the definition

$$
F_{\alpha \beta}=A_{\beta, \alpha}-A_{\alpha, \beta}
$$

the above two equations can be reduced to

$$
\frac{\partial A_{\phi}}{\partial t}=\frac{c^{2}}{4 \pi \sigma}\left(1-\frac{2 m}{r}\right)^{1 / 2} \sin \theta\left[\frac{\partial}{\partial r}\left\{\left(1-\frac{2 m}{r}\right) \frac{1}{\sin \theta} \frac{\partial A_{\phi}}{\partial r}\right\}+\frac{\partial}{\partial \theta}\left(\frac{1}{r^{2} \sin \theta} \frac{\partial A_{\phi}}{\partial \theta}\right)\right] .
$$

Choosing

$$
A_{\phi}=\frac{f(r, t)}{r} \sin \theta
$$


for the flat space-time and

$$
A_{\phi}=-g(r, t) \sin ^{2} \theta
$$

for the curved space-time, where $r$ and $\theta$ are the spherical radius and polar angle respectively one gets from equation (11) and equation (11)

$$
\frac{\partial^{2} f(x, t)}{\partial x^{2}}-\frac{2}{x^{2}} f(x, t)=\frac{4 \pi R^{2} \sigma}{c^{2}} \frac{\partial f(x, t)}{\partial t}
$$

and

$$
\left(1-\frac{y}{x}\right)^{1 / 2}\left[\left(1-\frac{y}{x}\right) \frac{\partial^{2} g(x, t)}{\partial x^{2}}+\frac{y}{x^{2}} \frac{\partial g(x, t)}{\partial x}-\frac{2}{x^{2}} g(x, t)\right]=\frac{4 \pi R^{2} \sigma}{c^{2}} \frac{\partial g(x, t)}{\partial t}
$$

respectively, where $x=r / R, R$ is the radius of the star and $y=2 m / R$.

For both relativistic and non-relativistic cases, I impose the usual boundary conditions as given in Urpin \& Muslimov (1992).

\section{THE MODEL}

I shall follow Urpin \& Van Riper (1993) in my approach. The formalisms adopted in the present work have been discussed in details by Urpin \& Muslimov (1992). The gravitational mass of the star and its radius are taken to be $1.4 M_{\odot}$ and $7.35 \mathrm{~km}$ respectively.

\subsection{The Initial Configurations}

If one assumes the initial value of $f(r, t)=f(r)$ at $t=0$ for flat space-time, then for curved space-time (Wasserman \& Shapiro 1983; Sengupta 1995)

$$
g(r, 0)=g(r)=\frac{3 r f(r)}{8 m^{3}}\left[r^{2} \ln \left(1-\frac{2 m}{r}\right)+2 m r+m^{2}\right] .
$$

This is because of the fact that any given magnetic field configuration in flat space-time is modified by the curvature of space-time produced by the gravitational field of the central object. Asymptotically at a large distance $g(r)$ coincides with $f(r)$. 
I have considered the decay of the magnetic field which initially occupies the surface layers of the crust up to a depth (i) $x=0.979$ which corresponds to the density $4 \times 10^{11} \mathrm{gm}$ $\mathrm{cm}^{-3}$ and (ii) $x=0.9834$ which corresponds to density $2 \times 10^{11} \mathrm{gm} \mathrm{cm}^{-3}$.

\subsection{The Electrical Conductivity}

The electrical conductivity within the crust has been calculated following the approaches of Urpin \& Van Riper (1993). The net conductivity of the crustal material at a given depth is computed as

$$
\sigma=\left(\frac{1}{\sigma_{p h}}+\frac{1}{\sigma_{i m p}}\right)^{-1},
$$

where $\sigma_{p h}$ is the conductivity due to electron-phonon scattering and $\sigma_{i m p}$ is the conductivity due to electron-impurity scattering. The effect of electron-ion scattering has been neglected since the region where this effect could be important is sufficiently thin. $\sigma_{i m p}$ is inversely proportional to the impurity parameter $Q$ defined as

$$
Q=\frac{1}{n} \sum_{i} n_{i}\left(Z-Z_{i}\right)^{2},
$$

where $n$ and $Z$ are the number density and electric charge of background ions in the crust lattice without impurity, $Z_{i}$ and $n_{i}$ are the charge and density of the $i$-th impurity species. The summation is extended over all species of impurity. It is worth mentioning that the value of the impurity parameter $Q$ for neutron star crust is not known at present. Electron-impurity scattering becomes more important with increasing density and decreasing temperature. Hence at the late stage of evolution when the temperature becomes low, the conductivity is dominated by electron-impurity scattering. The impurity parameter $Q$ has been taken as $0.0,0.005,0.05$ in the present work. The conductivity is calculated by assuming that the region under consideration is isothermal. This is justified because the thermal evolution calculations show that the crust becomes isothermal at an 
age between 1 and a 1000 yr. For the thermal evolution of the neutron star I adopt both the standard as well as the accelerated cooling models for neutron stars with gravitational mass $1.4 M_{\odot}$ and radius $7.35 \mathrm{~km}$ as used by Urpin and Van Riper (1993). The models are based on soft equation of state (Baym, Pethick \& Sutherland 1971) with normal npematter in the core and with standard neutrino emissivities for the slowly cooling case and with enhanced neutrino emission due to the Urca process on percolating quarks for the rapidly cooling case.

\section{RESULTS AND DISCUSSIONS}

Equations (14) and (15) with their corresponding boundary conditions have been solved numerically using the standard Crank-Nicholson differencing scheme. The results for Schwarzschild geometry have been transformed into a local Lorentz frame by using equation (7) and are compared with those for the flat space-time in order to visualize the effect of general relativity.

The evolution of the surface magnetic field normalized to its initial value for both the general relativistic and the non-relativistic cases with the standard cooling model and with the impurity parameter $Q=0$ are presented in Figure 1 . In this case, the electrical conductivity within the crust is determined by electron-phonon scattering only.

\section{EDITOR: PLACE FIGURE 1 HERE.}

It is shown by Muslimov \& Urpin (1992) that the field behavior is qualitatively independent of the forms of the initial configurations but the numerical results differ for

various choices of the initial depth penetrated by the magnetic field. At a very early stage of evolution when the crustal matter is melted in the layers of a maximal current density, 
the magnetic field does not decay appreciably. After the outer crust solidifies, significant decay takes place and after 1 Myr no decay occurs if the impurity content is zero.

The above scenario for flat space-time is not altered with the inclusion of general relativistic effects but significant decrease in the numerical value is found when the effect of space-time curvature is incorporated. Figure 1 shows that if the initial field configuration extends up to the density $\rho_{0}=4 \times 10^{11} \mathrm{gm} \mathrm{cm}^{-3}$, then the decay is much slower than that for $\rho_{0}=2 \times 10^{11} \mathrm{gm} \mathrm{cm}^{-3}$. This is due to the fact that in the former case, the currents were localized in deeper layers with higher conductivity from the very initial stage. Figure 1, 2 and 3 show that during the period when the electrical conductivity is mainly determined by the phonon-electron scattering, the decay rate, for the general relativistic case, of the

magnetic field initially penetrated up to a density $\rho_{0}=2 \times 10^{11} \mathrm{gm} \mathrm{cm}^{-3}$ is almost the same to that when the general relativistic effect is not included and the initial field is penetrated up to a higher density $\rho_{0}=4 \times 10^{11} \mathrm{gm} \mathrm{cm}^{-3}$. This implies that the curvature of space-time acts as if it pushes the currents towards a deeper layer of the crust.

\section{EDITOR: PLACE FIGURE 2 HERE.}

\section{EDITOR: PLACE FIGURE 3 HERE.}

Figure 2 and Figure 3 show the magnetic field decay with the standard cooling model and with the impurity parameter $Q=0.005$ and $Q=0.05$ respectively. The impurity-electron scattering is dominant at the late stage of evolution when the crustal temperature is low. As a consequence the decay rate after $t>10 \mathrm{Myr}$ changes appreciably. Figure 2 and Figure 3 show that the general relativistic effect tends to suppress the role of the impurity content that accelerate the decay rate. If the impurity parameter $Q$ is as high as 0.05 , then at the late stage the reduction in decay rate by the effect of space-time 
curvature is more than an order of magnitude. Thus, general relativistic effect is more important at the late stage of evolution of the crustal magnetic field.

Figure 4 and Figure 5 present the magnetic field decay with the accelerated cooling model and with the impurity parameter $Q=0.005$ and $Q=0.05$ respectively. If the accelerated cooling model is adopted for the description of the thermal evolution of the neutron star, then the decay rate even for the flat space-time is found to be negligible when the value of $Q$ is zero or very small, i.e., when the electron-phonon scattering is the only or the dominating process in determining the electrical conductivity within the crust. Therefore, the general relativistic effects in this situation is not interesting.

EDITOR: PLACE FIGURE 4 HERE.

\section{EDITOR: PLACE FIGURE 5 HERE.}

It is clear from the figures that the magnetic field decay is qualitatively different for the standard and the accelerated cooling models. During the neutrino cooling period the crustal temperature is much higher for the standard cooling model than that of the accelerated cooling case. This makes the electrical conductivity for the standard slowly cooling model lower than that for the accelerated cooling model. As a consequence, the decay in the magnetic field is less for the accelerated cooling case compared to that for the standard cooling model. Unlike the case for standard cooling model, the decay for the accelerated cooling model does not exhibit a flat region at 1 - 10 Myr. For the accelerated cooling model, noticeable decay occurs due to the electron-impurity scattering only and hence there is practically no decay if the impurity content is very small. However, if the value of the impurity parameter $Q$ is greater than say, $10^{-3}$ then after 10 Myr noticeable decay takes place. Figure 4 and Figure 5 demonstrate that general relativistic effect reduces 
the decay rate nearly by $50 \%$ even if the value of $Q$ is very high. This decrease in the decay rate is almost constant throughout the subsequent period of evolution. But if the impurity parameter $Q$ is as high as 0.05 , then there is a rapid increase in the decay rate after $10^{9} \mathrm{yr}$ for the flat space-time. The same type of increase in the decay rate is not found for the general relativistic case as the effect of the impurity content is suppressed by the effect of space-time curvature. Consequently, if the impurity content is very high, then after $10^{9}$ yr, the rate of decay reduces by more than one order of magnitude if one incorporates the general relativistic effects. However, from the consideration of the age of isolated neutron stars, this very late stage difference in the evolution of the magnetic field may not be relevant from the observational point of view. Nevertheless, a $50 \%$ reduction in the decay rate by the effect of general relativity alone is certainly important when accelerated cooling model is considered.

\section{CONCLUSIONS}

It is shown that the general relativistic effect plays a crucial role in determining the magnetic field decay within the crust of isolated neutron star and the importance of the effect is no less than that of the initial depth penetrated by the magnetic field. At the late stage of evolution, the effect of space-time curvature produced by the intense gravitational field of the star suppresses the role of the impurity content of the crustal material and thus reduces the rate of field decay substantially. If the neutron star cools rapidly due to the existence of quark matter in the core then the decay rate becomes much lower than that when standard slow cooling is considered. A further reduction in the decay rate by the effect of space-time curvature results such a small decay in the magnetic field (during the whole life span of the star) that irrespective of the uncertainties in the impurity content,

existence of quark matter that can give rise to accelerated cooling may possibly be ruled 
out if more than just two orders of magnitude decrease from the initial field strength is observationally inferred.

The author is thankful to the anonymous referee for constructive comments and useful suggestions. Thanks are also due to Bhaskar Datta for some discussion and Somnath Bharadwaj for a critical reading of the manuscript. 


\section{REFERENCES}

Bhattacharya, D., \& Datta, B. 1996, MNRAS, 282, 1059

Chanmugam, G. 1992, ARA\&A, 30,143

Chanmugam, G., \& Sang, Y. 1989, MNRAS, 241, 295

Lamb, F. 1991, in ASP Conf. Proc. 20, Frontiers of Stellar Evolution, ed. D. Lambert (San Francisco: ASP), 299

Phinney, S., \& Kulkarni, S. 1994, ARA\&A, 32, 591

Pethic, C. J., \& Sahrling, M. 1995, ApJ, 453, L29

Sang, Y., \& Chanmugam, G. 1987, ApJ, 323, L61

Sengupta, S. 1995, ApJ, 449, 224

Sengupta, S. 1997, ApJ, 479, L133

Urpin, V. A., \& Muslimov, A. G. 1992, MNRAS, 256, 261

Urpin, V. A., \& Van Riper, K. A. 1993, ApJ, 411, L87

Wakatsuki, S., Hikita, A., Sato, N., \& Itoh, N. 1992, ApJ, 392, 628

Wasserman, I., \& Shapiro, S. L. 1983, ApJ, 265, 1036 
Fig. 1. - The evolution of surface magnetic field normalized to its initial value for flat and curved space-times with the impurity parameter $Q=0$. Solid lines represent the results for curved space-time while broken lines represent that for flat space-time. The numbers near the curves indicate the value of $\rho_{0} / 10^{11} \mathrm{gm} \mathrm{cm}^{-3}$ where $\rho_{0}$ is the density corresponding to the initial depth penetrated by the magnetic field. The results are obtained by using the standard cooling model.

Fig. 2.- Same as figure 1 but with $Q=0.005$.

Fig. 3.- Same as figure 1 but with $Q=0.05$.

Fig. 4.- Same as figure 2 but with the accelerated cooling model.

Fig. 5.- Same as figure 3 but with the accelerated cooling model. 


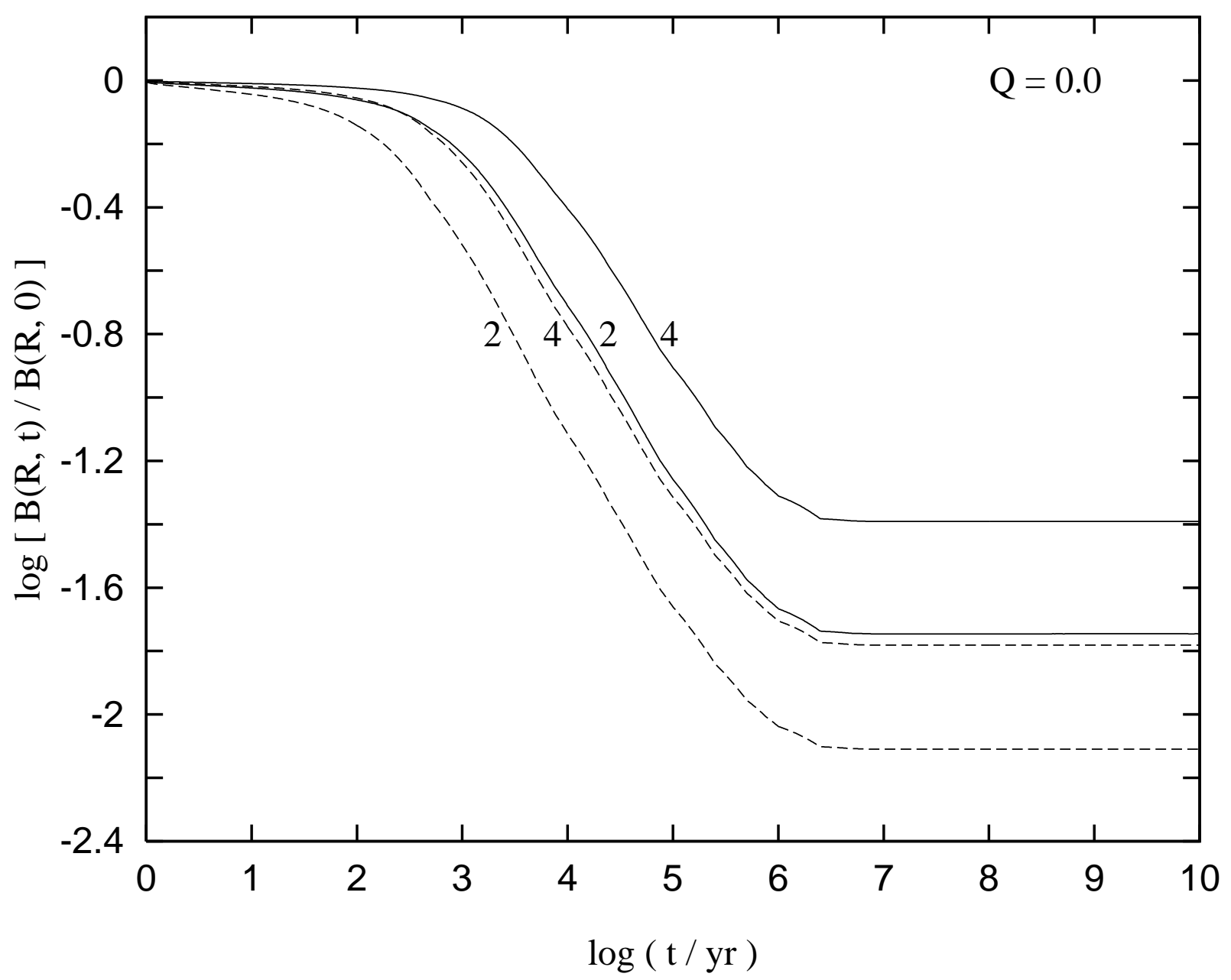




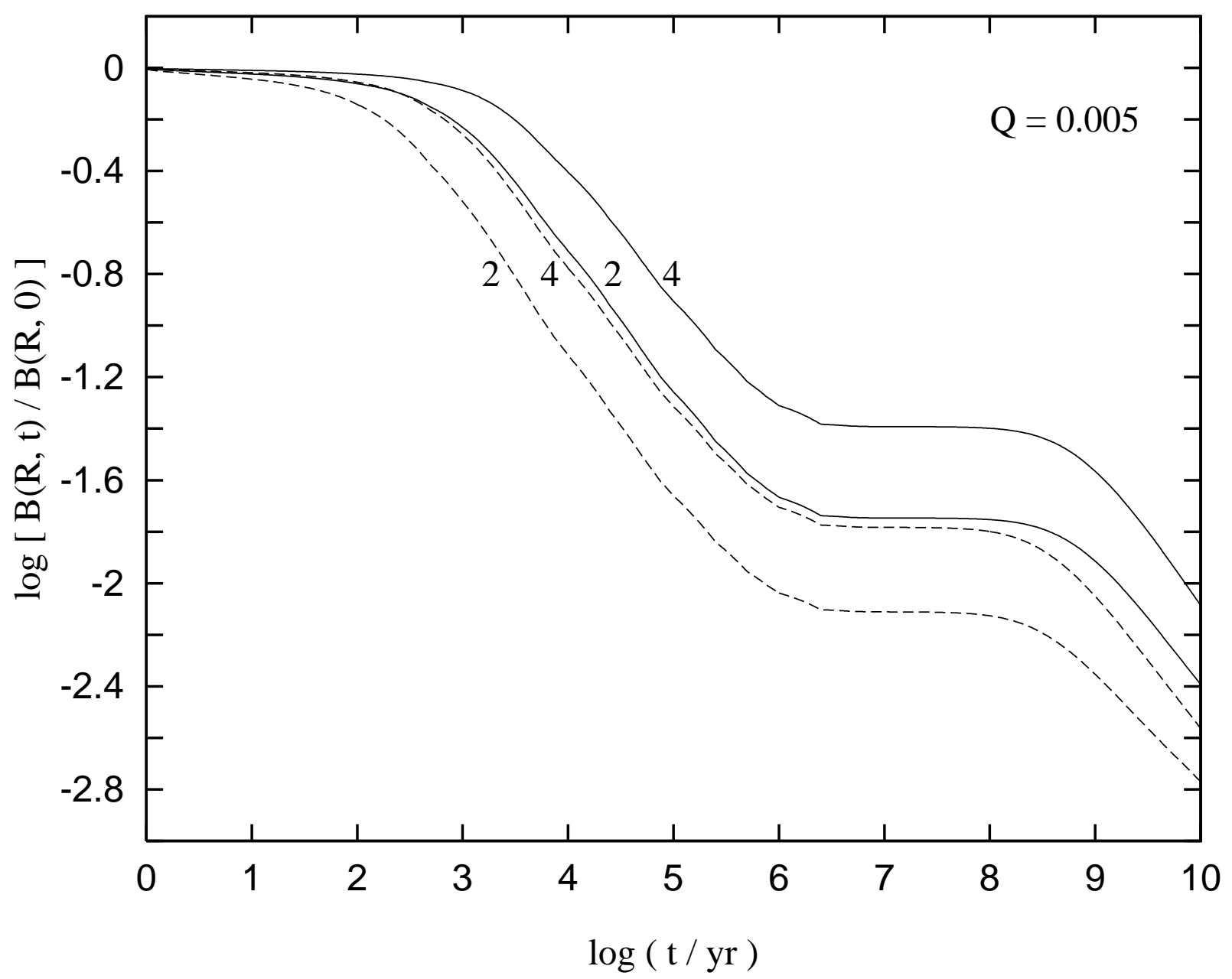




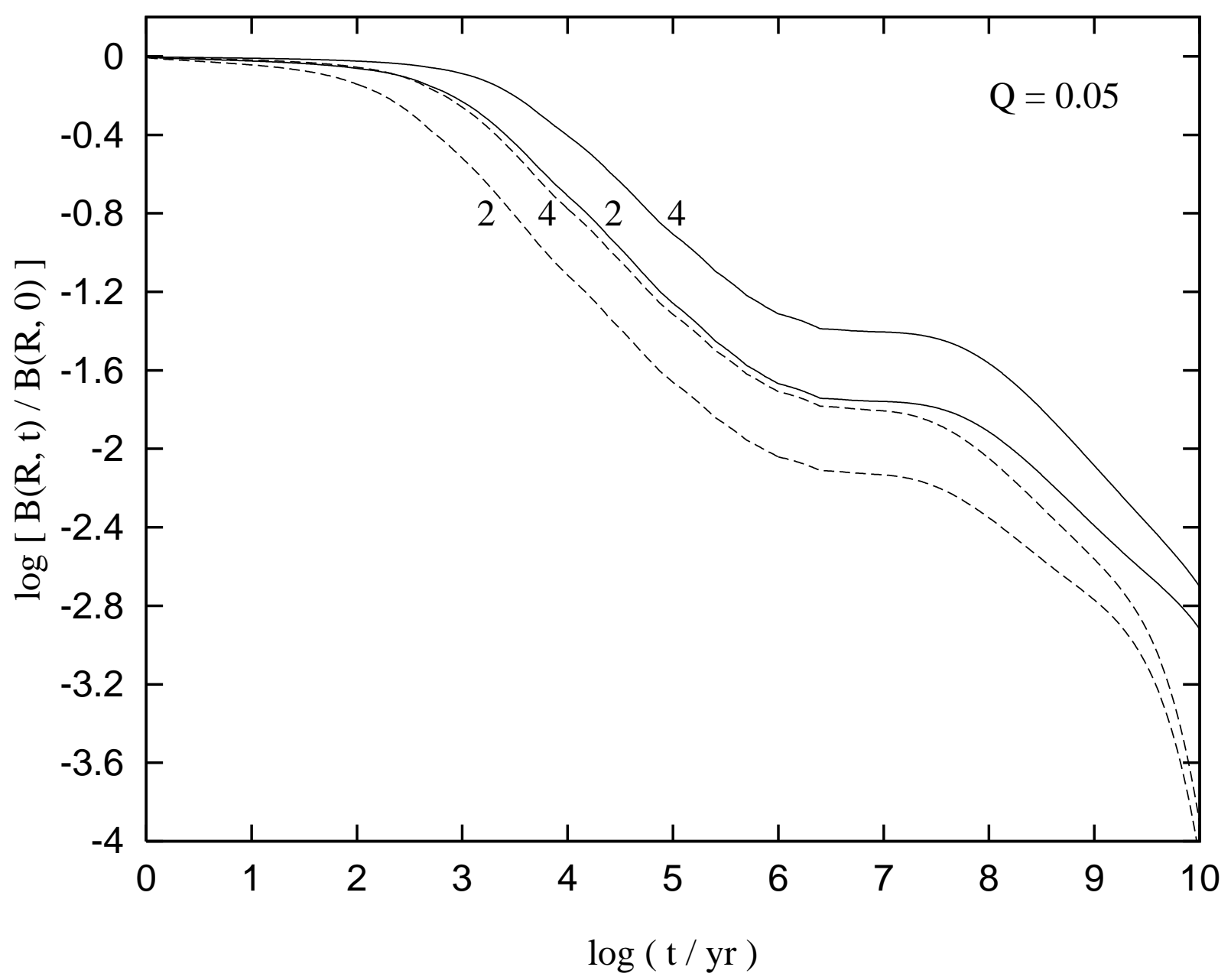




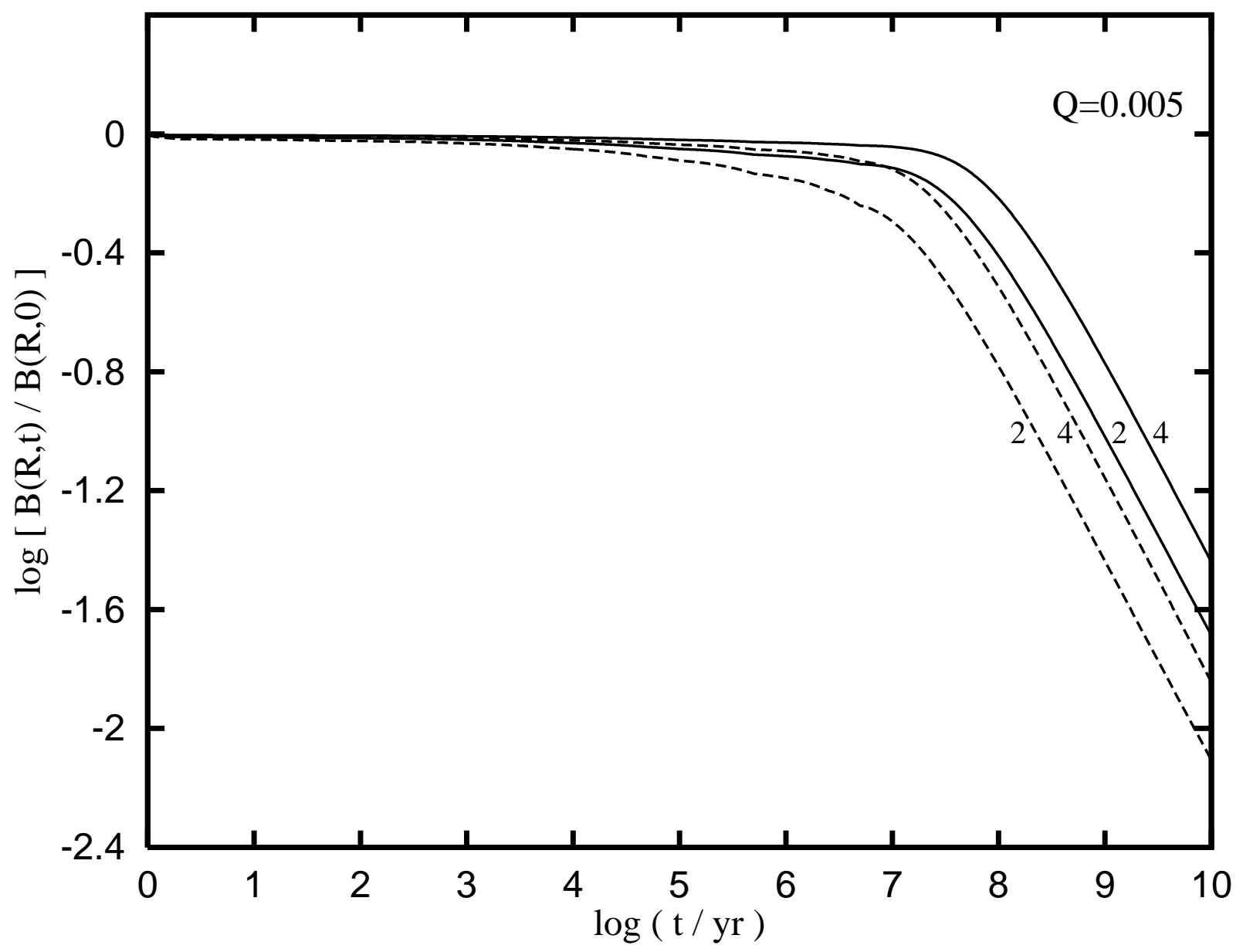




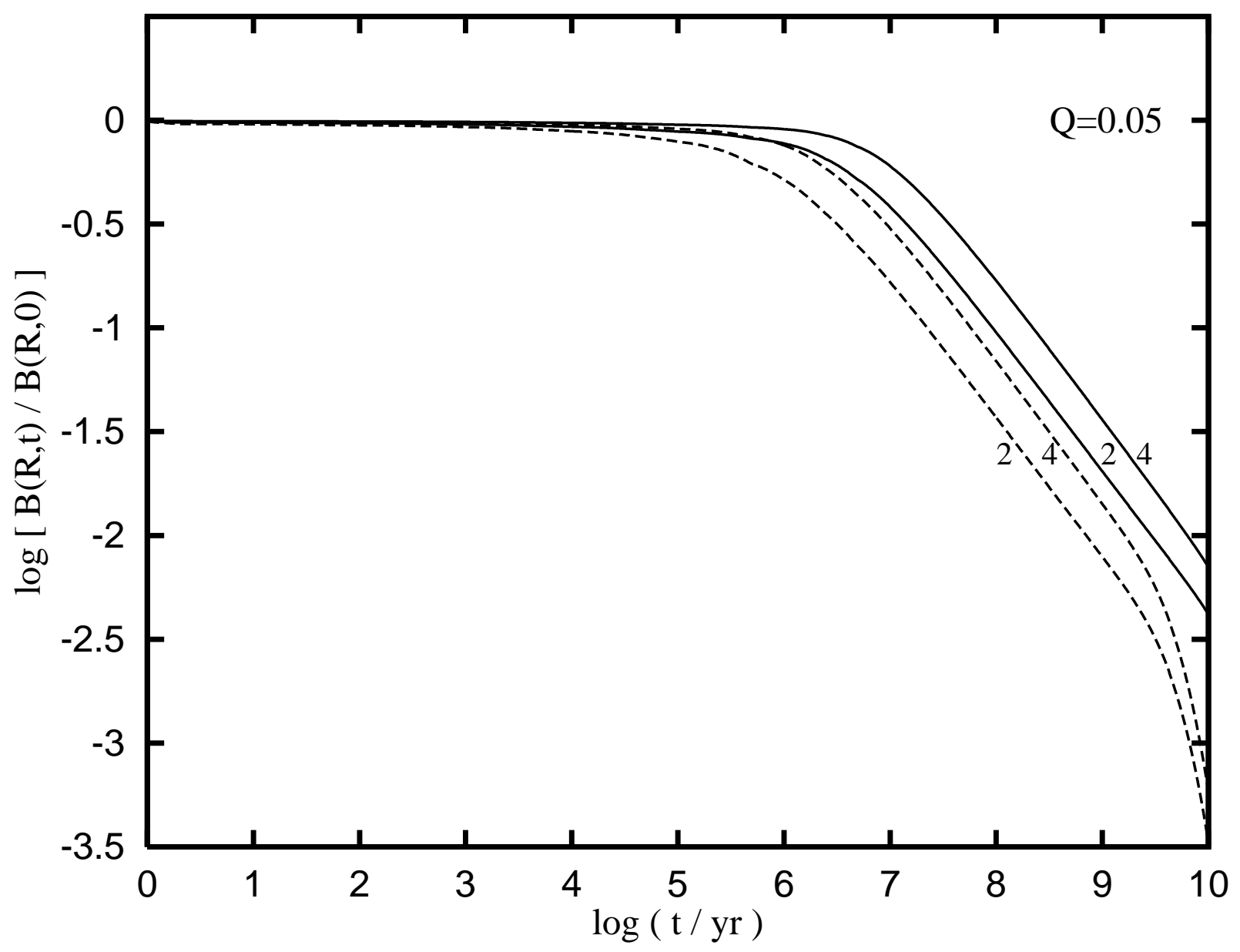

